EG Gstudios

Journal of Management and Economics for Iberoamerica

Artículo de investigación

\title{
Obstáculos del sector manufacturero ecuatoriano y argentino: evidencia empírica desde las empresas innovadoras
}

Silvana Astudillo*

Profesor-Investigador, Facultad de Ciencias de la Hospitalidad, Universidad de Cuenca, Cuenca, Ecuador.

silvana.astudillo@ucuenca.edu.ec

Anahí Briozzo

Profesor-Investigador, Departamento de Ciencias de la Administración, Universidad Nacional del Sur (UNS) e Instituto de Investigaciones Económicas y Sociales del Sur (UNS- CONICET), Bahía Blanca, Argentina.

abriozzo@uns.edu.ar

\section{Resumen}

Este trabajo presenta los obstáculos que perciben las empresas manufactureras de Argentina y Ecuador que innovaron en el producto y en el proceso en sus actividades empresariales. Se utilizó la base de datos de la Enterprise Surveys del Banco Mundial del 2017 a través de un análisis chi2 de Pearson y un modelo probit aplicados a una muestra de 103 empresas ecuatorianas y 644 empresas argentinas. La inestabilidad política, el financiamiento, las regulaciones laborales y la fuerza laboral inadecuada son limitaciones para las empresas argentinas. Los factores limitantes asociados a la innovación en el proceso son, en las empresas ecuatorianas, la corrupción; en el caso argentino son el crimen, el robo y el desorden y las regulaciones laborales.

Palabras clave: obstáculos; innovación en el producto; innovación en el proceso; Ecuador; Argentina.

\section{Obstacles of the Ecuadorian and Argentine manufacturing sector: empirical evidence from innovative enterprises}

\section{Abstract}

This paper presents the obstacles perceived by manufacturing enterprises in Argentina and Ecuador that innovated in the product and the process in their business activities. The 2017 World Bank Enterprise Surveys database was used applying a Pearson chi2 analysis and a probit model to a sample of 103 Ecuadorian enterprises and 644 Argentine enterprises. Political instability, financing, labor regulations, and inadequate labor force are constraints for Argentine enterprises. The limiting factors associated with innovation in the process are, in the Ecuadorian case, corruption in enterprises; in the Argentine case are crime, theft and disorder, and labor regulations.

Keywords: obstacles; product innovation; process innovation; Ecuador; Argentina.

\section{Obstáculos do setor manufatureiro equatoriano e argentino: evidência empírica de empresas inovadoras}

\section{Resumo}

Este artigo apresenta os obstáculos percebidos pelas empresas manufatureiras da Argentina e do Equador que inovaram no produto e no processo em suas atividades empresariais. Usou-se o banco de dados 2017 do Banco Mundial Enterprise Surveys por meio de uma análise de Qui-quadrado de Pearson e um modelo probit aplicados a uma amostra de 103 empresas equatorianas e 644 empresas argentinas. Instabilidade política, financiamento, regulamentações trabalhistas e uma força de trabalho inadequada são restrições para as empresas argentinas. Os fatores limitantes associados à inovação no processo são, nas empresas equatorianas, a corrupção; no caso argentino são o crime, o roubo e a desordem e as normas trabalhistas.

Palavras-chave: obstáculos; inovação de produto; inovação no processo; Equador; Argentina.

* Autor para dirigir correspondencia.

Clasificación JEL: 031; 032.

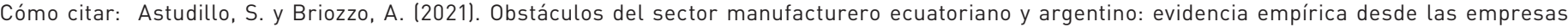
innovadoras. Estudios Gerenciales, 37(160), 387-398. https://doi.org/10.18046/j.estger.2021.160.4014

DOI: https://doi.org/10.18046/j.estger.2021.160.4014

Recibido: 7-may-2020

Aceptado: 3 - dic-2020

Publicado: 31-ago-2021 


\section{Introducción}

El reto de la innovación en el sector empresarial es un proceso que conlleva mucho esfuerzo. El entorno de la innovación en América Latina y el Caribe muestra un cuadro fragmentado y poco dinámico (De León y Fernández, 2016), y las tendencias observadas con respecto a los obstáculos para las empresas son similares entre estas regiones (Santiago, De Fuentes, Dutrénit y Gras, 2016). Dada la importancia de estudiar los factores que limitan la actividad innovadora, varios han sido los estudios que abordan las barreras de la innovación dentro de la actividad empresarial en países de Europa, Asia y América (Álvarez y Crespi, 2015; Santiago et al., 2016; Cecere, Corrocher y Mancusi, 2018). Sin embargo, son escasas las investigaciones comparativas sobre las limitantes de la actividad innovadora de las empresas entre países y en especial en Latinoamérica.

En este sentido, esta investigación compara los obstáculos - desde la percepción de las empresas innovadoras en el sector manufacturero en el producto y en el proceso- en dos países de la región: Argentina y Ecuador. Ambos países presentan diferentes perfiles de ciencia, tecnología e innovación (CTI), aunque con semejanzas en su desarrollo. Dutrénit (2012) manifiesta que Argentina pertenece a un grupo con un perfil alto dentro de la masa crítica de CTI y Ecuador presenta un desempeño inferior en la ciencia y en la innovación.

Según los datos de la Red de Indicadores de Ciencia y Tecnología Iberoamericana e Interamericana (RICYT, 2018), el gasto en investigación y desarrollo (I+D) con relación al producto interno bruto (PIB) argentino en 2016 fue del 0,53\%, y del $0,44 \%$ con relación al PIB ecuatoriano en 2014. Con respecto al número de personas involucradas en $\mathrm{I}+\mathrm{D}$, se registraron 79.333 para Argentina y 8948 para Ecuador, en los mismos años del caso anterior, respectivamente. Así mismo, el recurso humano con doctorado en el área de ciencia y tecnología registra 234 argentinos y 15 ecuatorianos. El coeficiente de invención, que comprende las patentes solicitadas por los residentes, es de 1,27 para el caso argentino y de 0,23 para el ecuatoriano.

Por lo tanto, esta investigación constituye un avance en la literatura empírica sobre la comparabilidad regional de las limitaciones de la innovación. De hecho, la comparación de Ecuador con un país de la región, en este caso Argentina, permite visualizar los retos y desafíos del país con respecto a sus medidas en materia de innovación dentro de su sistema institucional.

El trabajo de investigación plantea las siguientes preguntas: ¿cuáles son las percepciones de las empresas que innovaron en el producto y en el proceso del sector manufacturero ecuatoriano y argentino sobre los obstáculos de la actividad empresarial? ¿Difieren estos obstáculos de los percibidos por las empresas no innovadoras? ¿Los obstáculos de estas empresas ocurren en función de la innovación en el producto y en el proceso y por variables de la estructura de la empresa?

Después de esta introducción, se presenta una breve revisión de la literatura sobre los obstáculos de la innovación. Luego, se describe la metodología y los resultados. Finalmente, se resumen algunas conclusiones e implicaciones de los resultados.

\section{Revisión de la literatura}

En los últimos años, se registran esfuerzos crecientes para mejorar el entorno empresarial, que se reflejan al reducir las barreras del mercado para las empresas (Hoang-Nam y Bao-Tram, 2019). Sin embargo, el escenario de negocios en el que opera una empresa está condicionado por un conjunto de factores que impiden su actividad empresarial y disminuyen su propensión a innovar (Crespi, Olivari y Vargas 2016). Así pues, analizar las barreras de innovación es importante en los países para lograr un mayor crecimiento (Lundvall, 2016).

Algunos autores agrupan los condicionantes de la innovación en factores. Mohnen y Röller (2005) señalan que las barreras de la innovación se enmarcan en (a) la financiación, (b) en la falta de personal calificado, (c) en la falta de oportunidades de cooperación con otras empresas e instituciones tecnológicas y (d) en la legislación, las normas y los impuestos.

Así también, el Manual del Oslo clasifica las barreras de la innovación en cuatros factores: costos, conocimiento, institucionales y mercado (Organización para la Cooperación y el Desarrollo Económicos [OCDE], 2005). Por su parte, Sabato y Botana (2011) plantean el predominio de barreras en los aspectos sociocultural, financiero, político y científico. A su vez, Crespi et al. (2016) agrupan los obstáculos de la innovación en (a) costos, (b) factores de conocimiento, (c) factores de mercado y (d) factores relacionados con la apropiabilidad y otros.

La literatura investiga también los limitantes de la innovación de manera individual; en lo que respecta a los factores del mercado y los costos, las restricciones financieras reducen la innovación (Álvarez y Crespi, 2015; Bartels, Koria y Andriano, 2016; Cecere et al., 2018). Asimismo, los costos de producción son un limitante de la actividad innovadora (Coad, Pellegrino y Savona, 2015). A esto se suma la competencia, que puede influir negativamente en los incentivos de las empresas formales para innovar y adoptar nuevas tecnologías (Perry et al., 2007; Crespi et al., 2016).

En el caso de los factores políticos y macroeconómicos, la corrupción sugiere un impacto negativo en la actividad innovadora de las empresas IXu y Yano 2016; Paunov, 2016; Goedhuys, Mohnen y Taha 2016; Barasa, 2018; Pirtea, Sipos e Ionescu, 
2019). La inestabilidad política manifiesta un impacto negativo en la I+D y obstaculiza el intercambio del conocimiento y la innovación (Allard, Martínez y Williams, 2012; Masino, 2015). Sin embargo, es menos grave entre los empresarios que tienen conexiones políticas y aseguran el apoyo gubernamental para la inversión en innovación (Cumming, Rui y Wu, 2016). Por su parte, en Latinoamérica y en el Caribe, el marco institucional no fomenta efectivamente la innovación (Mohan, Watson y Strobl, 2016).

Los obstáculos reglamentarios, como las tasas impositivas, las regulaciones laborales y las licencias y permisos, son desincentivos para los empresarios (Kantis, Federico, Angelelli e Ibarra-García, 2016). En consecuencia, la falta de políticas gubernamentales representa una barrera formal para la innovación.

Dentro de los factores internos que obstaculizan la innovación, se encuentran los agrupados en el conocimiento. Un nivel de educación inadecuado en el capital humano es un obstáculo para la innovación (Botric y Božić, 2018; Petelski, Milesi y Verre, 2019). Otro argumento señala que las empresas admiten poseer un equipo humano bien cualificado, pero limitado para acometer procesos de innovación (Iglesias-Sánchez, Jambrino-Maldonado y De las Heras-Pedrosa, 2017).

Otros factores que se suman como obstáculos para la innovación son las telecomunicaciones, que requieren progresos y una infraestructura moderna y competitiva (López, Niembro y Ramos, 2017). De este modo, los países presentan obstáculos en la asequibilidad tecnológica, económica y confiabilidad tecnológica (Katz y Galperín, 2013). El transporte y la innovación también se relacionan; por ejemplo, las innovaciones implican introducir nueva logística, es decir, transporte, almacenaje y manejo de productos (OCDE y Eurostat, 2018) y sus dificultades se traducen en mayores costos para los productos que se trasladan directamente al consumidor (Sanabria, 2008).

Por otro lado, algunos autores manifiestan que el tamaño de la empresa se relaciona con los obstáculos de la innovación (Stendahl y Roos, 2008; de-Oliveira y Rodil-Marzábal, 2019). Existen también diferencias regionales en la percepción de los obstáculos (lammarino, Sanna-Randaccio y Savona, 2009). Con respecto a la antigüedad de la empresa, las empresas maduras otorgan mayor importancia a los obstáculos de la innovación con respecto a las empresas jóvenes (Pellegrino, 2018). De hecho, existe una relación negativa entre la edad de la empresa y las barreras de la innovación (Pellegrino, 2015).

En el caso argentino, las condiciones de financiación se manifiestan como una limitación (Edwards-Schachter, Castro- Martínez y Fernándezde-Lucio, 2011; Guercio, Vigier y Briozzo, 2014; Arza y López, 2018). Así también los obstáculos institucionales reducen la innovación (Lachman y López, 2019). Por otra parte, Petelski et al. (2019) señalan que el principal obstáculo de las actividades de innovación en las empresas manufactureras argentinas es la falta de personal calificado. También, el Foro Económico Mundial (WEF, 2016) señala que los factores más sobresalientes que dificultan los negocios son la inflación, los impuestos, el acceso al financiamiento, la corrupción, la burocracia ineficiente del gobierno, la inestabilidad política, entre otros. Con respecto al tamaño de la empresa, no se encuentran evidencias del efecto de los obstáculos de la innovación en esta variable en las empresas argentinas (Arza y López, 2018).

En el caso ecuatoriano, Zamora y Villamar (2011) manifiestan que los obstáculos que hacen que una empresa manufacturera no innove en ese país son las limitaciones de orden económico, la capacitación del personal, la falta de información para acceder a programas de fomento, servicios, centros de desarrollo e incentivos fiscales. Con respecto a las limitaciones de la actividad innovadora por su tamaño, las grandes empresas ecuatorianas perciben con menor intensidad los obstáculos (de-Oliveira y Rodil-Marzábal, 2019). Guaipatin y Schwartz (2014) señalan la inestabilidad política como la primera limitación empresarial en Ecuador. Por su parte, la WEF (2016) señala que los factores que obstaculizan los negocios en este país corresponden primordialmente a las regulaciones laborales, al acceso al financiamiento, los impuestos, entre otros. Asimismo, el Global Entrepreneurship Monitor del 2019 señala que las principales debilidades del emprendimiento en Ecuador son las políticas de gobierno relacionadas con impuestos y burocracia, el apoyo financiero y la transferencia de investigación y desarrollo (Lasio, Amaya, Zambrano y Ordeñana, 2019).

\section{Metodología}

\subsection{Universo y muestra}

El universo del estudio son las micro, pequeñas, medianas y grandes empresas manufactureras de Argentina y Ecuador. "El Banco Mundial toma una muestra estratificada siguiendo tres criterios: ubicación geográfica, tamaño de la empresa según el número de empleados, y sector de actividad" (Banco Mundial, 2014 , p. 1). La submuestra empleada en este estudio está compuesta por 103 empresas ecuatorianas y 644 empresas argentinas.

En la tabla 1 se identifica que el sector manufacturero más representativo en Argentina es el de alimentos y el mayor número de empresas se concentra en la pequeña empresa, de 10 a 49 empleados. Las empresas innovadoras corresponden 
a 318 en el producto y a 241 en el proceso. El mayor número de empresas manufactureras innovadoras en el producto y en el proceso se encuentra en Buenos Aires. Los subsectores manufactureros que más innovan en el producto son metales básicos, productos químicos, confecciones y madera. En la innovación en el proceso, se observan los subsectores metales básicos e instrumentos de precisión y publicaciones e impresiones.

Tabla 1. Argentina: distribución de la muestra

\begin{tabular}{|c|c|c|c|}
\hline Variables & $\begin{array}{l}\text { Porcentaje } \\
\text { del total }\end{array}$ & $\begin{array}{l}\text { Innovación } \\
\text { en el producto } \\
\text { (\% de firmas) }\end{array}$ & $\begin{array}{l}\text { Innovación } \\
\text { en el proceso } \\
\text { (\% de firmas) }\end{array}$ \\
\hline \multicolumn{4}{|l|}{ Tamaño de la empresa } \\
\hline Microempresa & 28,58 & 46,11 & 39,76 \\
\hline Pequeña & 51,23 & 58,59 & 46,33 \\
\hline Mediana & 17,76 & 40,76 & 41,11 \\
\hline Grande & 2,43 & 56,04 & 39,08 \\
\hline Total & $100 \%$ & & \\
\hline \multicolumn{4}{|l|}{ Subsector Manufactura } \\
\hline Alimentos & 23,41 & 37,92 & 32,92 \\
\hline Textiles & 4,42 & 57,74 & 50,43 \\
\hline Confecciones & 7,43 & 73,53 & 59,57 \\
\hline Cuero & 0,52 & 39,84 & 30,96 \\
\hline Madera & 0,32 & 70,38 & 8,63 \\
\hline Papel & 4,03 & 44,31 & 34,34 \\
\hline $\begin{array}{l}\text { Publicaciones, } \\
\text { impresiones }\end{array}$ & 0,96 & 9,38 & 73,00 \\
\hline Productos Químicos & 12,65 & 74,13 & 63,43 \\
\hline Plásticos y Cauchos & 7,99 & 31,53 & 23,31 \\
\hline $\begin{array}{l}\text { Productos minerales } \\
\text { no metálicos }\end{array}$ & 0,24 & 41,62 & 46,59 \\
\hline Metales Básicos & 0,55 & 100,00 & 8,18 \\
\hline $\begin{array}{l}\text { Fabricación de } \\
\text { productos metálicos }\end{array}$ & 12,50 & 55,39 & 52,65 \\
\hline Maquinaria y equipos & 15,88 & 50,69 & 48,58 \\
\hline Electrónicos & 2,71 & 68,00 & 19,91 \\
\hline $\begin{array}{l}\text { Instrumentos de } \\
\text { Precisión }\end{array}$ & 1,21 & 18,72 & 77,73 \\
\hline $\begin{array}{l}\text { Maquinarias de } \\
\text { Transporte }\end{array}$ & 3,33 & 36,85 & 14,01 \\
\hline Otros & 1,84 & 88,87 & 52,86 \\
\hline \multicolumn{4}{|l|}{ Provincia } \\
\hline Buenos Aires & 80,08 & 52,73 & 44,93 \\
\hline Rosario & 6,06 & 52,17 & 37,91 \\
\hline Mendoza & 4,75 & 58,76 & 40,37 \\
\hline Córdoba & 6,44 & 41,80 & 30,98 \\
\hline Tucumán & 2,67 & 51,69 & 35,70 \\
\hline
\end{tabular}

Nota: para las estimaciones se utilizaron las ponderaciones del muestreo aleatorio estratificado con el comando svy de STATA.

Fuente: elaboración propia con base en la Enterprise Survey del Banco Mundial (2017).

En la tabla 2 se identifica que el sector manufacturero más representativo en Ecuador corresponde al de alimentos, mientras que el mayor número de empresas se concentra en la pequeña empresa, de 10 a 49 empleados. Las empresas manufactureras innovadoras corresponden a 90 en el producto y a 61 en el proceso. La mayoría de las firmas innovadoras se ubica en Pichincha. Las actividades manufactureras que más innovan en el producto son textiles, alimentos, confecciones y productos metálicos. En la innovación en el proceso, se observa confecciones y textiles, productos metálicos y alimentos.

Tabla 2. Ecuador: distribución de la muestra

\begin{tabular}{|c|c|c|c|}
\hline Variables & $\begin{array}{l}\text { Porcentaje } \\
\text { del total }\end{array}$ & $\begin{array}{l}\text { Innovación } \\
\text { en el producto } \\
\text { (\% de firmas) }\end{array}$ & $\begin{array}{l}\text { Innovación } \\
\text { en el proceso } \\
\text { (\% de firmas) }\end{array}$ \\
\hline \multicolumn{4}{|l|}{ Tamaño de la empresa } \\
\hline Microempresa & 10,33 & 96,70 & 78,74 \\
\hline Pequeña & 53,11 & 85,58 & 58,87 \\
\hline Mediana & 25,29 & 84,63 & 50,75 \\
\hline Grande & 11,27 & 97,95 & 79,19 \\
\hline Total & $100 \%$ & & \\
\hline \multicolumn{4}{|c|}{ Actividad manufacturera } \\
\hline Alimentos & 18,04 & 94,85 & 77,67 \\
\hline Textiles & 5,35 & 100 & 73,12 \\
\hline Confecciones & 8,77 & 87,09 & 88,05 \\
\hline Cuero & 2,33 & 80,95 & 76,79 \\
\hline Madera & 5,43 & 64,12 & 49,67 \\
\hline Papel & 3,76 & 15,51 & 73,84 \\
\hline $\begin{array}{l}\text { Publicaciones, } \\
\text { impresiones }\end{array}$ & 7,47 & 83,61 & 35,66 \\
\hline Productos Químicos & 10,00 & 81,76 & 59,27 \\
\hline Plásticos y Cauchos & 11,53 & 100 & 76,09 \\
\hline $\begin{array}{l}\text { Productos minerales } \\
\text { no metálicos }\end{array}$ & 6,10 & 90,06 & 9,94 \\
\hline $\begin{array}{l}\text { Fabricación de } \\
\text { productos metálicos }\end{array}$ & 11,59 & 91,98 & 82,27 \\
\hline Maquinaria y equipos & 2,75 & 79,64 & 26,05 \\
\hline Otras & 6,88 & 100 & 50,00 \\
\hline \multicolumn{4}{|l|}{ Provincia } \\
\hline Pichincha & 71,75 & 89,10 & 59,80 \\
\hline Guayas & 24,59 & 80,61 & 66,55 \\
\hline Azuay & 3,66 & 83,41 & 51,45 \\
\hline
\end{tabular}

Nota: para las estimaciones se utilizaron las ponderaciones del muestreo aleatorio estratificado con el comando svy de STATA.

Fuente: elaboración propia con base en la Enterprise Survey del Banco Mundial (2017).

\subsection{Modelo econométrico}

Para los propósitos de la investigación, se utilizó la información de la Enterprise Survey del Banco Mundial del año 2017', que señala solamente las variables innovación en el producto e innovación en el proceso como medidas cualitativas de la innovación, ambas resultado de la percepción de los empresarios. Para efectos de esta investigación, se

1 La encuesta empresarial del Banco Mundial para Argentina y Ecuador (2017) proporciona datos recopilados desde marzo de 2017 hasta marzo de 2018, que es la última encuesta empresarial que proporciona el Banco Mundial para ambos países. 
toman los conceptos de las variables innovación en el producto y la innovación en el proceso expuestos en el Manual del Oslo: “la innovación de producto corresponde con la introducción de un bien o servicio nuevo o significativamente mejorado, en cuanto a sus características o en cuanto al uso que se destina" (OCDE, 2005, p. 56). A su vez, la innovación en el proceso es "la introducción de un nuevo, o significativamente mejorado, proceso de producción o de distribución. Ello implica cambios significativos en las técnicas, los materiales y/o programas informáticos" (OCDE, 2005, p. 59).

En la investigación se utilizó un análisis bivariado a través de la prueba de Pearson chi2 para determinar si los obstáculos difieren según la empresa sea o no innovadora (tablas 3 y 4). Dos variables categóricas (como empresa innovadora y tipo de obstáculol se consideran independientes si todas las probabilidades conjuntas son iguales al producto de las probabilidades marginales. En este caso, la hipótesis nula sería, por ejemplo, que el porcentaje de empresas innovadoras es igual para la percepción de un determinado obstáculo, la cual se rechaza si el $p$-value es inferior al nivel crítico (por ejemplo 5\%). La prueba Pearson chi2 se calcula como:

$$
\chi^{2}=\sum_{i=1}^{n} \frac{\left(o_{i}-E_{i}\right)^{2}}{E_{i}}=N \sum_{i=1}^{n} p i\left(\frac{\frac{o_{i}}{N}-p_{i}}{p_{i}}\right)^{2}
$$

Donde

$X^{2}=$ valor del estadístico.

$O_{i}=$ número de observaciones del tipo $i$.

$N=$ número total de observaciones.

$E_{i}=N p_{i}=$ la frecuencia esperada del tipo $i$, derivada de la hipótesis nula de que la fracción de $i$ en la población es $p_{i}$.

$\mathrm{n}=$ número de celdas en la tabla.

Además, para la etapa de análisis multivariado, se planteó un modelo de respuesta binaria probit, que se estima según la siguiente ecuación:

$$
P(y=1 \mid x)=G\left(\beta_{0}+\beta_{1} x_{1}+\ldots+\beta_{k} x_{k}\right)
$$

Donde $G$ es la función de distribución acumulada de la normal estándar:

$G(z)=\Phi(z)=\int_{-\infty}^{z} \phi(v) d v$ y $\phi(z)$ es la densidad de la normal estándar. La variable dependiente es por naturaleza binaria, definida como:

$y=1$ si la empresa identifica un determinado factor como obstáculo.

$y=0$ en caso contrario.
La estimación del modelo se realiza mediante máxima verosimilitud, y las estimaciones tienen la propiedad de ser asintóticamente normales, consistentes y eficientes. El efecto que tiene el cambio en una variable sobre la probabilidad del resultado puede estimarse a través del cambio parcial o marginal en $\operatorname{Pr}(y=1 \mid \mathrm{X})$. Dado:

$$
\operatorname{Pr}(y=1 \mid \mathrm{X})=F(\mathrm{X} \beta)
$$

el cambio marginal se computa como:

$$
\frac{\partial \operatorname{Pr}(y=1 \mid \mathrm{X})}{\partial x_{k}}=\frac{\partial F(\mathrm{X} \beta)}{\partial x_{k}}=f(\mathrm{X} \beta) \beta_{k}
$$

Donde f(.) es la función de densidad de $F($.).

De esta forma, la probabilidad de que un obstáculo de la actividad empresarial manufacturera ocurra (tablas 5 y 6) se estima en función de un conjunto de regresores: si la empresa innovó en el producto o en el proceso (variable explicatival y un grupo de variables control que captan la estructura de la empresa, como el tamaño, la antigüedad, la ubicación geográfica y el subsector. Las variables dependientes e independientes para el estudio se definen en la tabla 3. Asimismo, para las estimaciones se utilizó el comando svy de STATA, con el fin de considerar los pesos muestrales.

\section{Resultados}

\subsection{Análisis bivariado}

En el caso de Argentina, la tabla 4 muestra que los principales obstáculos para el empresariado manufacturero son la administración tributaria, la inestabilidad política y las regulaciones laborales. Desde un análisis estadístico descriptivo, los obstáculos con mayor porcentaje desde la percepción de las empresas manufactureras que innovan en el producto son el crimen, robo y desorden, el transporte, la inestabilidad política y la regulación aduanera y comercial. Con respecto a las empresas que innovan en el proceso, la percepción de obstáculos presenta porcentajes altos en crimen, robo y desorden, y en el transporte.

Al analizar la hipótesis nula de si la presencia de obstáculos es independiente de la actividad innovadora, los resultados fueron significativos en las barreras de la actividad empresarial, como regulaciones laborales y fuerza laboral con una educación inadecuada, lo que revela que estas dificultades no son independientes de la actividad innovadora. De igual manera, en la innovación en el proceso, los obstáculos corrupción y crimen, robo y desorden resultaron significativos, por lo que no son independientes de la actividad innovadora. 
Tabla 3. Ecuador y Argentina: variables dependientes e independientes

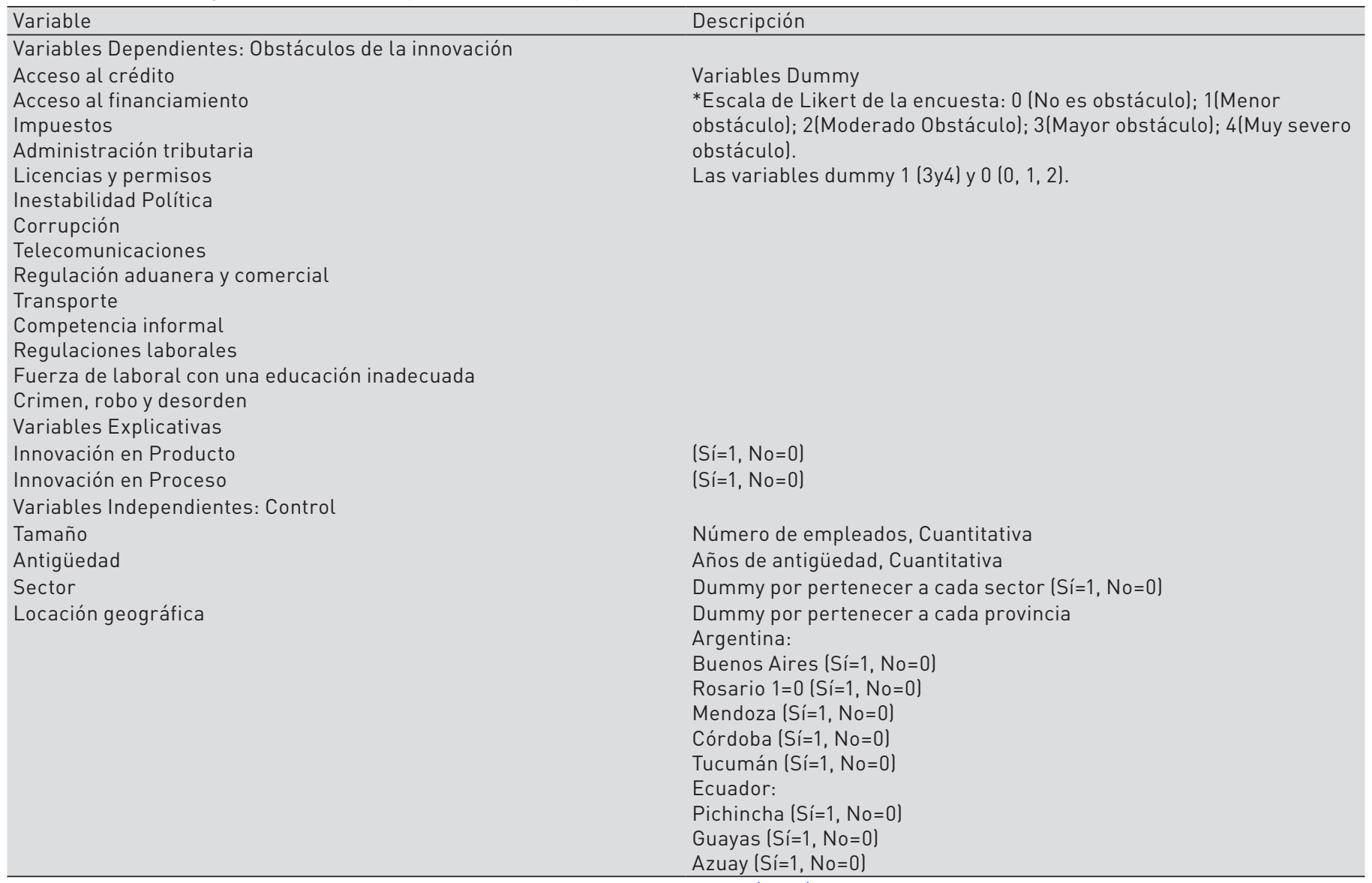

Fuente: elaboración propia con base en la Enterprise Survey del Banco Mundial (2017).

Tabla 4. Argentina: diferencia entre empresas innovadoras y no innovadoras

\begin{tabular}{|c|c|c|c|c|c|}
\hline Obstáculos & $\begin{array}{c}\text { Percepción del } \\
\text { obstáculo } \\
\text { Porcentaje de firmas } \\
\end{array}$ & $\begin{array}{c}\text { Innovación en el Producto } \\
\text { Porcentaje de Firmas } \\
\text { del obstáculo }\end{array}$ & $\begin{array}{c}\text { Chi2 } \\
\text { p-valor }\end{array}$ & $\begin{array}{c}\text { Innovación en el proceso } \\
\text { Porcentaje de Firmas } \\
\text { del obstáculo }\end{array}$ & $\begin{array}{c}\text { Chi2 } \\
\text { p-valor }\end{array}$ \\
\hline Acceso al financiamiento & 27,88 & 45,63 & 0,3540 & 48,22 & 0,3629 \\
\hline Licencias y Permisos & 14,32 & 49,97 & 0,8760 & 35,85 & 0,4192 \\
\hline Inestabilidad Política & 54,38 & 54,03 & 0,5205 & 38,07 & 0,1136 \\
\hline Corrupción & 41,40 & 47,24 & 0,2813 & 34,10 & $0,0524^{*}$ \\
\hline Transporte & 23,59 & 58,90 & 0,2303 & 50,15 & 0,2646 \\
\hline Competencia informal & 37,77 & 51,25 & 0,8151 & 38,47 & 0,3092 \\
\hline Regulaciones laborales & 52,29 & 44,87 & $0,0538^{*}$ & 43,13 & 0,2749 \\
\hline Fuerza laboral con una educación inadecuada & 34,96 & 38,64 & $0,0095^{*}$ & 39,75 & 0,5822 \\
\hline Crimen, robo y desorden & 13,10 & 60,06 & 0,3832 & 61,34 & $0,0580^{*}$ \\
\hline
\end{tabular}

Nota: ${ }^{*} 10 \%$ de significancia.

Fuente: elaboración propia con base en la Enterprise Survey del Banco Mundial (2017).

Respecto a Ecuador, la tabla 5 evidencia que los principales obstáculos para el empresariado manufacturero son la inestabilidad política y la corrupción. A través de un análisis estadístico descriptivo, las empresas manufactureras que innovan en el producto señalan con porcentajes altos las limitaciones regulación aduanera y comercial y el transporte. Con respecto a las empresas manufactureras que innovan en el proceso, la percepción de los obstáculos presenta porcentajes altos en crimen, robo y desorden, acceso al financiamiento y la corrupción.

Al analizar la hipótesis nula de si la presencia de obstáculos es independiente de la actividad innovadora, los resultados no fueron significativos, lo que revela que los obstáculos de la actividad empresarial son independientes de la actividad innovadora, es decir, la percepción de las limitaciones es la misma para las empresas innovadoras y no innovadoras. 
Tabla 5. Ecuador: diferencia entre empresas innovadoras y no innovadoras

\begin{tabular}{|c|c|c|c|c|c|}
\hline Obstáculos & $\begin{array}{l}\text { Percepción del } \\
\text { obstáculo } \\
\text { Porcentaje del } \\
\text { Total firmas }\end{array}$ & $\begin{array}{c}\text { Innovación en el } \\
\text { Producto } \\
\text { Porcentaje de Firmas } \\
\text { del obstáculo }\end{array}$ & $\begin{array}{c}\text { Chi2 } \\
\text { p-valor }\end{array}$ & $\begin{array}{c}\text { Innovación en el } \\
\text { proceso } \\
\text { Porcentaje de Firmas } \\
\text { del obstáculo }\end{array}$ & $\begin{array}{l}\text { Chi2 } \\
\text { p-valor }\end{array}$ \\
\hline Administración Tributaria & 39,50 & 80,80 & 0,2772 & 47,18 & 0,1141 \\
\hline Licencias y Permisos & 32,90 & 80,63 & 0,3380 & 58,82 & 0,7539 \\
\hline Telecomunicaciones & 25,98 & 91,97 & 0,3593 & 58,17 & 0,7742 \\
\hline Regulación aduanera y comercial & 25,5 & 94,07 & 0,2066 & 54,70 & 0,5802 \\
\hline Transporte & 16,04 & 91,13 & 0,5545 & 57,44 & 0,7840 \\
\hline Competencia informal & 36,88 & 87,04 & 0,9603 & 63,58 & 0,7872 \\
\hline
\end{tabular}

Fuente: elaboración propia con base en la Enterprise Survey del Banco Mundial (2017).

\subsection{Análisis multivariado}

Las tablas 6 y 7 muestran los resultados del modelo de regresión probit, que estima la probabilidad de que los obstáculos de la actividad empresarial en Argentina y en Ecuador se presenten en función de la actividad innovadora en el producto y en el proceso.

En la tabla 6, con respecto a la innovación en el producto, Argentina presenta que las barreras acceso al financiamiento, regulaciones laborales y fuerza laboral con una educación inadecuada son menos probables de ser percibidas en las empresas del sector manufacturero. La inestabilidad política presenta una significancia estadística positiva con la innovación en el producto, por lo que las empresas innovadoras en el producto tienen más probabilidad de presentar esta limitación. No obstante, el nivel de significancia es del $90 \%$.

El obstáculo crimen, robo y desorden presenta una significancia estadística positiva con la innovación en el proceso, lo que revela que las empresas innovadoras en el proceso son más propensas a evidenciar esta limitación. A su vez, las regulaciones laborales son significantes; sin embargo, es menos probable que sean percibidas en empresas que innovan en el proceso.

Por otro lado, el análisis multivariado de las variables de la estructura de la empresa señala que las provincias de Buenos Aires y Rosario son menos propensas a la presencia del obstáculo acceso al financiamiento. La limitación administración tributaria tiene una significancia positiva con el sector alimentos.

La provincia de Córdoba es más propensa a presentar la limitación licencias y permisos.
La barrera inestabilidad política presenta una significancia positiva con el subsector maquinaria de transportes, lo que revela que este sector tiene más probabilidad de percibir esta limitación. Sin embargo, existe una significancia estadística negativa con las provincias de Buenos Aires, Mendoza y Rosario; es decir, es menos probable que estas provincias presenten esta limitación. Con referencia a telecomunicaciones, la provincia de Mendoza tiene más probabilidad de presentar esta barrera y el subsector maquinaria de transporte, menos probabilidad. La regulación aduanera y comercial tiene significancia estadística negativa con la provincia de Rosario.

Con respecto a la corrupción, Buenos Aires, Rosario, Mendoza y los subsectores minerales y eléctricos son menos propensos a presentar este obstáculo. Buenos Aires, Mendoza y Córdoba son menos propensas a presentar la barrera transporte. En la limitación fuerza laboral con una educación inadecuada, Buenos Aires es menos propensa a percibir este obstáculo, así como en los subsectores de papel e instrumentos. En la barrera crimen, robo y desorden, las provincias de Buenos Aires y Mendoza son menos propensas a percibir este obstáculo. Las variables tamaño y antigüedad no resultaron significativas en la estimación.

Para Ecuador, la tabla 7 evidencia que la corrupción presenta una significancia estadística positiva con la innovación en el proceso, lo que revela que a medida que las empresas innovan en el proceso aumenta la propensión a percibir que la corrupción es un obstáculo para su desarrollo. La innovación en el producto no presenta significancia estadística en la estimación. 
Tabla 6. Argentina: modelo probit de los obstáculos de la actividad innovadora

\begin{tabular}{|c|c|c|c|c|c|c|c|c|c|c|c|c|}
\hline $\begin{array}{l}\text { Variables } \\
\text { Dependientes }\end{array}$ & $\begin{array}{c}\text { Acceso } \\
\text { al } \\
\text { financiamiento }\end{array}$ & $\begin{array}{l}\text { Administración } \\
\text { Tributaria }\end{array}$ & $\begin{array}{c}\text { Licencias } \\
y \\
\text { Permisos }\end{array}$ & $\begin{array}{l}\text { Inestabilidad } \\
\text { Política }\end{array}$ & Corrupción & $\begin{array}{l}\text { Tele- } \\
\text { comunicaciones }\end{array}$ & $\begin{array}{l}\text { Regulación } \\
\text { Aduanera y } \\
\text { comercial }\end{array}$ & Transporte & $\begin{array}{l}\text { Competencia } \\
\text { informal }\end{array}$ & $\begin{array}{c}\text { Regulaciones } \\
\text { Laborales }\end{array}$ & $\begin{array}{l}\text { Fuerza de } \\
\text { trabajo } \\
\text { inadecuada }\end{array}$ & $\begin{array}{c}\text { Crimen, } \\
\text { robo, } \\
\text { desorden }\end{array}$ \\
\hline \multicolumn{13}{|c|}{ Variables Explicativas } \\
\hline $\begin{array}{l}\text { Innovación en } \\
\text { el producto }\end{array}$ & $\begin{array}{c}0,071^{*} \\
(-0,221)\end{array}$ & $\begin{array}{c}0,509 \\
(0,075)\end{array}$ & $\begin{array}{c}0,333 \\
(0,153)\end{array}$ & $\begin{array}{l}0,100^{*} \\
(0,212)\end{array}$ & $\begin{array}{c}0,349 \\
(-1,073)\end{array}$ & $\begin{array}{c}0,701 \\
(0,043)\end{array}$ & $\begin{array}{c}0,838 \\
(0,028)\end{array}$ & $\begin{array}{c}0,725 \\
(0,041)\end{array}$ & $\begin{array}{c}0,176 \\
(-0,159)\end{array}$ & $\begin{array}{c}0,086^{*} \\
(-0,196)\end{array}$ & $\begin{array}{l}0,001 * * \\
(-0,405)\end{array}$ & $\begin{array}{c}0,920 \\
(0,018)\end{array}$ \\
\hline $\begin{array}{l}\text { Innovación en } \\
\text { el proceso }\end{array}$ & $\begin{array}{c}0,248 \\
(0,145)\end{array}$ & $\begin{array}{c}0,525 \\
(-0,074)\end{array}$ & $\begin{array}{c}0,981 \\
(-0,003)\end{array}$ & $\begin{array}{c}0,393 \\
(-0,100)\end{array}$ & $\begin{array}{c}0,101 \\
(-0,192)\end{array}$ & $\begin{array}{c}0,751 \\
(0,036)\end{array}$ & $\begin{array}{c}0,394 \\
(0,122)\end{array}$ & $\begin{array}{c}0,639 \\
(0,057)\end{array}$ & $\begin{array}{c}0,447 \\
(0,092)\end{array}$ & $\begin{array}{c}0,094^{*} \\
(-0,194)\end{array}$ & $\begin{array}{c}0,929 \\
(-0,010)\end{array}$ & $\begin{array}{l}0,008^{*} \\
(0,388)\end{array}$ \\
\hline \multicolumn{13}{|c|}{ Variables Control } \\
\hline Tamaño & $\begin{array}{c}0,559 \\
(-0,000)\end{array}$ & $\begin{array}{c}0,124 \\
(0,000)\end{array}$ & $\begin{array}{c}0,767 \\
(0,000)\end{array}$ & $\begin{array}{c}0,489 \\
(-0,000)\end{array}$ & $\begin{array}{c}0,174 \\
(-0,000)\end{array}$ & $\begin{array}{c}0,710 \\
(-0,000)\end{array}$ & $\begin{array}{c}0,932 \\
(0,000)\end{array}$ & $\begin{array}{c}0,758 \\
(0,000)\end{array}$ & $\begin{array}{c}0,791 \\
(-0,000)\end{array}$ & $\begin{array}{c}0,684 \\
(0,000)\end{array}$ & $\begin{array}{c}0,892 \\
(-0,000)\end{array}$ & $\begin{array}{c}0,213 \\
(-0,000)\end{array}$ \\
\hline Antigüedad & $\begin{array}{c}0,471 \\
(-0,002)\end{array}$ & $\begin{array}{c}0,371 \\
(-0,002)\end{array}$ & $\begin{array}{c}0,985 \\
(-0,000)\end{array}$ & $\begin{array}{c}0,207 \\
(0,003)\end{array}$ & $\begin{array}{c}0,222 \\
(0,003)\end{array}$ & $\begin{array}{c}0,579 \\
(-0,000)\end{array}$ & $\begin{array}{c}0,117 \\
(0,005)\end{array}$ & $\begin{array}{c}0,291 \\
(-0,003)\end{array}$ & $\begin{array}{c}0,726 \\
(-0,001)\end{array}$ & $\begin{array}{c}0,280 \\
(-0,003)\end{array}$ & $\begin{array}{c}0,521 \\
(-0,001)\end{array}$ & $\begin{array}{c}0,499 \\
(-0,002)\end{array}$ \\
\hline Buenos Aires & $\begin{array}{c}0,080^{*} \\
(-0,386)\end{array}$ & $\begin{array}{c}0,993 \\
(0,002)\end{array}$ & $\begin{array}{c}0,116 \\
(0,428)\end{array}$ & $\begin{array}{l}0,001 * * \\
(-0,786)\end{array}$ & $\begin{array}{c}0,000^{*} \\
(-0,975)\end{array}$ & $\begin{array}{c}0,772 \\
(-0,061)\end{array}$ & $\begin{array}{c}0,315 \\
(-0,239)\end{array}$ & $\begin{array}{c}0,014^{*} \\
(-0,532)\end{array}$ & $\begin{array}{c}0,981 \\
(0,005)\end{array}$ & $\begin{array}{c}0,501 \\
(-0,145)\end{array}$ & $\begin{array}{c}0,029 * \\
(-0,468)\end{array}$ & $\begin{array}{c}0,029^{*} \\
(-0,548)\end{array}$ \\
\hline Rosario & $\begin{array}{c}0,069^{*} \\
(-0,451)\end{array}$ & $\begin{array}{c}0,993 \\
(0,221)\end{array}$ & $\begin{array}{c}0,225 \\
(0,368)\end{array}$ & $\begin{array}{c}0,001^{*} \\
(-0,824)\end{array}$ & $\begin{array}{c}0,000^{*} \\
(-0,971)\end{array}$ & $\begin{array}{c}0,626 \\
(0,115)\end{array}$ & $\begin{array}{c}0,074^{*} \\
(-0,500)\end{array}$ & $\begin{array}{c}0,499 \\
(-0,161)\end{array}$ & $\begin{array}{c}0,487 \\
(-0,171)\end{array}$ & $\begin{array}{c}0,376 \\
(-0,211)\end{array}$ & $\begin{array}{c}0,176 \\
(-0,322)\end{array}$ & $\begin{array}{c}0,580 \\
(-0,151)\end{array}$ \\
\hline Mendoza & $\begin{array}{c}0,177 \\
(-0,330)\end{array}$ & $\begin{array}{c}0,361 \\
(0,221)\end{array}$ & $\begin{array}{c}0,158 \\
(0,429)\end{array}$ & $\begin{array}{l}0,086^{* *} \\
(-0,439]\end{array}$ & $\begin{array}{c}0,001^{*} \\
(-0,835)\end{array}$ & $\begin{array}{l}0,099 * \\
(0,395)\end{array}$ & $\begin{array}{c}0,332 \\
(-0,260)\end{array}$ & $\begin{array}{c}0,049 * \\
(-0,477)\end{array}$ & $\begin{array}{c}0,596 \\
(0,129)\end{array}$ & $\begin{array}{c}0,593 \\
(-0,129)\end{array}$ & $\begin{array}{c}0,441 \\
(0,183)\end{array}$ & $\begin{array}{c}0,032^{*} \\
(-0,626)\end{array}$ \\
\hline Córdoba & $\begin{array}{c}0,286 \\
(-0,260)\end{array}$ & $\begin{array}{c}0,987 \\
(0,003)\end{array}$ & $\begin{array}{l}0,007 * \\
(0,793)\end{array}$ & $\begin{array}{c}0,163 \\
(-0,344)\end{array}$ & $\begin{array}{c}0,160 \\
(-0,363)\end{array}$ & $\begin{array}{c}0,495 \\
(-0,161)\end{array}$ & $\begin{array}{c}0,135 \\
(-0,398)\end{array}$ & $\begin{array}{c}0,015^{*} \\
(-0,596)\end{array}$ & $\begin{array}{c}0,439 \\
(-0,188)\end{array}$ & $\begin{array}{c}0,643 \\
(-0,110)\end{array}$ & $\begin{array}{c}0,151 \\
(-0,339)\end{array}$ & $\begin{array}{c}0,155 \\
(-0,311)\end{array}$ \\
\hline Alimentos & $\begin{array}{c}0,730 \\
(0,164)\end{array}$ & $\begin{array}{l}0,073^{*} \\
(0,793)\end{array}$ & $\begin{array}{c}0,459 \\
(0,444)\end{array}$ & $\begin{array}{c}0,429 \\
(-0,331)\end{array}$ & $\begin{array}{c}0,412 \\
(-0,380)\end{array}$ & $\begin{array}{c}0,489 \\
(-0,310)\end{array}$ & $\begin{array}{c}0,572 \\
(-0,272)\end{array}$ & $\begin{array}{c}0,198 \\
(0,619)\end{array}$ & $\begin{array}{c}0,971 \\
(-0,015)\end{array}$ & $\begin{array}{c}0,698 \\
(0,167)\end{array}$ & $\begin{array}{c}0,863 \\
(0,076)\end{array}$ & $\begin{array}{c}0,466 \\
(0,194)\end{array}$ \\
\hline $\begin{array}{l}\text { Maquinaria } \\
\text { de Transporte }\end{array}$ & $\begin{array}{c}0,931 \\
(0,340)\end{array}$ & $\begin{array}{c}0,243 \\
(0,606)\end{array}$ & $\begin{array}{c}0,593 \\
(0,361)\end{array}$ & $\begin{array}{l}0,089 * \\
(0,881)\end{array}$ & $\begin{array}{c}0,698 \\
(-0,210)\end{array}$ & $\begin{array}{c}0,065^{*} \\
(-0,974)\end{array}$ & $\begin{array}{c}0,553 \\
(0,329)\end{array}$ & $\begin{array}{c}0,877 \\
(0,086)\end{array}$ & $\begin{array}{c}0,391 \\
(-0,456)\end{array}$ & $\begin{array}{c}0,455 \\
(-0,375)\end{array}$ & $\begin{array}{c}0,274 \\
(0,561)\end{array}$ & $\begin{array}{c}0,355 \\
(0,365)\end{array}$ \\
\hline Minerales & $\begin{array}{c}0,953 \\
(0,055)\end{array}$ & $\begin{array}{c}0,243 \\
(0,236)\end{array}$ & $\begin{array}{c}0,356 \\
(0,696)\end{array}$ & $\begin{array}{c}0,936 \\
(-0,047)\end{array}$ & $\begin{array}{l}0,026 * * \\
(-1,464)\end{array}$ & $\begin{array}{c}0,522 \\
(-0,384)\end{array}$ & $\begin{array}{c}0,982 \\
(0,015)\end{array}$ & $\begin{array}{c}0,147 \\
(0,918)\end{array}$ & $\begin{array}{c}0,813 \\
(0,139)\end{array}$ & $\begin{array}{c}0,355 \\
(-0,549)\end{array}$ & $\begin{array}{c}0,614 \\
(-0,314)\end{array}$ & $\begin{array}{c}0,693 \\
(-0,248)\end{array}$ \\
\hline Eléctricos & $\begin{array}{c}0,976 \\
(0,019)\end{array}$ & $\begin{array}{c}0,961 \\
(0,023)\end{array}$ & $\begin{array}{c}0,961 \\
(0,040)\end{array}$ & $\begin{array}{c}0,400 \\
(0,509)\end{array}$ & $\begin{array}{c}0,051^{*} \\
(-1,283)\end{array}$ & $\begin{array}{c}0,166 \\
(-0,870)\end{array}$ & $\begin{array}{c}0,982 \\
(0,015)\end{array}$ & $\begin{array}{c}0,631 \\
(-0,349)\end{array}$ & $\begin{array}{c}0,653 \\
(0,214)\end{array}$ & $\begin{array}{c}0,587 \\
(0,331)\end{array}$ & $\begin{array}{c}0,571 \\
(0,101)\end{array}$ & - \\
\hline Papel & $\begin{array}{c}0,257 \\
(0,654)\end{array}$ & $\begin{array}{c}0,391 \\
(0,470)\end{array}$ & $\begin{array}{c}0,400 \\
(0,592)\end{array}$ & $\begin{array}{c}0,386 \\
(0,470)\end{array}$ & $\begin{array}{c}0,116 \\
(-0,912)\end{array}$ & $\begin{array}{c}0,316 \\
(-0,586)\end{array}$ & $\begin{array}{c}0,635 \\
(0,285)\end{array}$ & $\begin{array}{c}0,954 \\
(0,553)\end{array}$ & $\begin{array}{c}0,496 \\
(-0,380)\end{array}$ & $\begin{array}{c}0,554 \\
(-0,322)\end{array}$ & $\begin{array}{l}0,079 * \\
(0,979)\end{array}$ & - \\
\hline Instrumentos & $\begin{array}{c}0,874 \\
(-0,125)\end{array}$ & $\begin{array}{c}0,791 \\
(0,189)\end{array}$ & $\begin{array}{c}0,350 \\
(0,779)\end{array}$ & $\begin{array}{c}0,973 \\
(5,129)\end{array}$ & $\begin{array}{c}0,634 \\
(0,385)\end{array}$ & $\begin{array}{c}0,557 \\
(0,466)\end{array}$ & $\begin{array}{c}0,152 \\
(1,055)\end{array}$ & $\begin{array}{c}0,985 \\
(0,014)\end{array}$ & $\begin{array}{c}0,234 \\
(0,946)\end{array}$ & $\begin{array}{c}0,271 \\
(0,858)\end{array}$ & $\begin{array}{l}0,071^{*} \\
(1,441)\end{array}$ & - \\
\hline
\end{tabular}

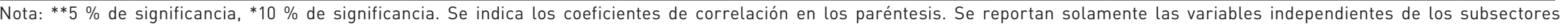
manufactureros que son significantes en el modelo. Tucumán en la Argentina presenta omisión en los valores.

Fuente: elaboración propia con base en la Enterprise Survey del Banco Mundial (2017). 
Tabla 7. Ecuador: modelo probit de los obstáculos de la actividad innovadora

\begin{tabular}{|c|c|c|c|c|c|c|c|c|c|c|c|c|}
\hline $\begin{array}{l}\text { Variables } \\
\text { Dependientes }\end{array}$ & $\begin{array}{c}\text { Acceso } \\
\text { al } \\
\text { financiamiento }\end{array}$ & $\begin{array}{l}\text { Administración } \\
\text { Tributaria }\end{array}$ & $\begin{array}{l}\text { Licencias y } \\
\text { Permisos }\end{array}$ & $\begin{array}{c}\text { Inestabilidad } \\
\text { Política }\end{array}$ & Corrupción & $\begin{array}{l}\text { Tele- } \\
\text { comunicaciones }\end{array}$ & $\begin{array}{c}\text { Regulación } \\
\text { Aduanera y } \\
\text { comercial }\end{array}$ & Transporte & $\begin{array}{l}\text { Competencia } \\
\text { informal }\end{array}$ & $\begin{array}{c}\text { Regulaciones } \\
\text { Laborales }\end{array}$ & $\begin{array}{c}\text { Fuerza de } \\
\text { trabajo } \\
\text { inadecuada }\end{array}$ & $\begin{array}{l}\text { Crimen, } \\
\text { robo, } \\
\text { desorden }\end{array}$ \\
\hline \multicolumn{13}{|c|}{ Variables Explicativas } \\
\hline $\begin{array}{l}\text { Innovación en } \\
\text { el producto }\end{array}$ & $\begin{array}{c}0,734 \\
(0,225)\end{array}$ & $\begin{array}{c}0,128 \\
(-0,746)\end{array}$ & $\begin{array}{c}0,223 \\
(-0,738)\end{array}$ & $\begin{array}{c}0,941 \\
(0,037)\end{array}$ & $\begin{array}{c}0,586 \\
(0,268)\end{array}$ & $\begin{array}{c}0,308 \\
(0,586)\end{array}$ & $\begin{array}{c}0,139 \\
(0,935)\end{array}$ & $\begin{array}{c}0,169 \\
(1,100)\end{array}$ & $\begin{array}{c}0,708 \\
(0,186)\end{array}$ & $\begin{array}{c}0,432 \\
(-0,360)\end{array}$ & $\begin{array}{c}0,325 \\
(0,666)\end{array}$ & $\begin{array}{c}0,985 \\
(6,753)\end{array}$ \\
\hline $\begin{array}{l}\text { Innovación en } \\
\text { el proceso }\end{array}$ & $\begin{array}{c}0,905 \\
(-0,515)\end{array}$ & $\begin{array}{c}0,219 \\
(-0,405)\end{array}$ & $\begin{array}{c}0,988 \\
(0,005)\end{array}$ & $\begin{array}{c}0,222 \\
(0,388)\end{array}$ & $\begin{array}{l}0,007 * \\
(0,893)\end{array}$ & $\begin{array}{l}(0,349) \\
(-0,321)\end{array}$ & $\begin{array}{c}0,530 \\
(0,239)\end{array}$ & $\begin{array}{c}0,701 \\
(-0,163)\end{array}$ & $\begin{array}{c}0,496 \\
(0,221)\end{array}$ & $\begin{array}{c}0,992 \\
(-0,003)\end{array}$ & $\begin{array}{c}0,645 \\
(-0,170)\end{array}$ & $\begin{array}{c}0,966 \\
(-0,017)\end{array}$ \\
\hline \multicolumn{13}{|c|}{ Variables control } \\
\hline Tamaño & $\begin{array}{c}0,614 \\
(0,000)\end{array}$ & $\begin{array}{c}0,796 \\
(0,000)\end{array}$ & $\begin{array}{c}0,189 \\
(-0,001)\end{array}$ & $\begin{array}{c}0,292 \\
(-0,000)\end{array}$ & $\begin{array}{c}0,143 \\
(-0,001)\end{array}$ & $\begin{array}{c}0,817 \\
(0,000)\end{array}$ & $\begin{array}{c}0,542 \\
(-0,000)\end{array}$ & $\begin{array}{c}0,993 \\
(0,000)\end{array}$ & $\begin{array}{c}0,278 \\
(-0,001)\end{array}$ & $\begin{array}{c}0,365 \\
(0,000)\end{array}$ & $\begin{array}{c}0,384 \\
(-0,001)\end{array}$ & $\begin{array}{c}0,130 \\
(-0,003)\end{array}$ \\
\hline Antigüedad & $\begin{array}{c}0,886 \\
(0,016)\end{array}$ & $\begin{array}{c}0,873 \\
(0,001)\end{array}$ & $\begin{array}{c}0,633 \\
(0,000)\end{array}$ & $\begin{array}{c}0,176 \\
(0,109)\end{array}$ & $\begin{array}{c}0,600 \\
(0,004)\end{array}$ & $\begin{array}{c}0,488 \\
(-0,006)\end{array}$ & $\begin{array}{c}0,732 \\
(0,003)\end{array}$ & $\begin{array}{c}0,120 \\
(-0,025)\end{array}$ & $\begin{array}{c}0,982 \\
(-0,000)\end{array}$ & $\begin{array}{c}0,403 \\
(0,006)\end{array}$ & $\begin{array}{c}0,514 \\
(-0,006)\end{array}$ & $\begin{array}{c}0,863 \\
(0,002)\end{array}$ \\
\hline Pichincha & $\begin{array}{l}0,034^{*} \\
(1,350)\end{array}$ & $\begin{array}{c}0,114 \\
(0,734)\end{array}$ & $\begin{array}{l}0,028^{*} \\
(1,530)\end{array}$ & $\begin{array}{l}0,090^{* *} \\
(0,717)\end{array}$ & $\begin{array}{c}0,719 \\
(0,156)\end{array}$ & $\begin{array}{l}0,021^{*} \\
(1,507)\end{array}$ & $\begin{array}{l}0,036 * \\
(1,446)\end{array}$ & $\begin{array}{c}0,454 \\
(0,3,79)\end{array}$ & $\begin{array}{c}0,314 \\
(-0,428)\end{array}$ & $\begin{array}{c}0,250 \\
(0,525)\end{array}$ & $\begin{array}{c}0,488 \\
(0,335)\end{array}$ & $\begin{array}{c}0,932 \\
(-0,037)\end{array}$ \\
\hline Guayas & - & $\begin{array}{c}0,536 \\
(0,358)\end{array}$ & $\begin{array}{c}0,874 \\
(0,125)\end{array}$ & $\begin{array}{c}0,424 \\
(-0,410)\end{array}$ & $\begin{array}{c}0,558 \\
(-0,301)\end{array}$ & $\begin{array}{l}0,029 * \\
(1,600)\end{array}$ & $\begin{array}{c}0,689 \\
(0,325)\end{array}$ & - & $\begin{array}{c}0,075^{*} \\
(-0,970)\end{array}$ & $\begin{array}{c}0,785 \\
(-1,523)\end{array}$ & $\begin{array}{c}0,825 \\
(0,130)\end{array}$ & - \\
\hline
\end{tabular}

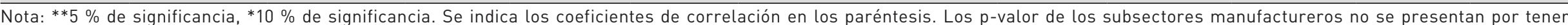
significancia estadística. La provincia del Azuay presenta omisión en los valores.

Fuente: elaboración propia con base en la Enterprise Survey del Banco Mundial (2017). 
A su vez, el análisis de las variables de la estructura de la empresa señala que las barreras acceso al financiamiento, licencias y permisos, inestabilidad política, telecomunicaciones y regulaciones aduaneras y comerciales tienen una significancia estadística positiva con la provincia de Pichincha, lo que revela que esta provincia es más propensa a presentar estas limitaciones.

El obstáculo competencia informal presenta una significancia negativa en la provincia del Guayas, lo que evidencia que las empresas manufactureras provenientes de esta provincia son menos propensas a presentar este obstáculo. La barrera telecomunicaciones es positiva con la provincia del Guayas, esta provincia es más propensa a presentar esta barrera. El tamaño, la antigüedad y los subsectores manufactureros no presentan significancia estadística.

\section{Conclusiones e implicaciones}

El objetivo de la investigación fue comparar los obstáculos de la actividad empresarial desde la percepción de las empresas innovadoras en el producto y en el proceso del sector manufacturero argentino y ecuatoriano con base en la Enterprise Survey del Banco Mundial del año 2017.

Con respecto a los resultados de investigación en el caso ecuatoriano, las empresas del sector manufacturero que innovan en los procesos son más propensas a presentar como limitación la corrupción; en la innovación en el producto no existen factores limitantes positivos asociados.

En el caso de las empresas del sector manufacturero argentino que innovan en el producto, se evidencia mayor probabilidad de presentar como barrera la inestabilidad política, que coincide con la investigación de Masino (2015) en su estudio en países miembros de la OCDE, y en la innovación en el proceso destacan el crimen, el robo y el desorden. Con menor propensión se evidencia en empresas argentinas que innovan en el producto barreras como el acceso al financiamiento, las regulaciones laborales y la fuerza de trabajo con educación inadecuada; estas limitaciones coinciden con hallazgos de investigaciones realizadas en Argentina (Guercio et al., 2014; WEF, 2016; Arza y López, 2018; Petelski et al., 2019).

Se evidenció una propensión similar de la innovación del proceso en ambos sectores manufactureros a las limitaciones asociadas a los factores políticos e institucionales de ambos países: corrupción, inestabilidad política y crimen y robo. Los hallazgos coinciden con lo planteado por Masino (2015); Xu y Yano (2016); Paunov (2016); Goedhuys et al. (2016); Barasa (2018); Pirtea et al. (2019), que consideran que la corrupción y la inestabilidad política impactan negativamente en la innovación de sectores empresariales en diferentes países alrededor del mundo.

El análisis de la información también revela que la antigüedad de la empresa y el tamaño son variables independientes de las barreras de la actividad empresarial en ambos sectores, contrario a lo señalado por Stendahl y Roos, 2008; de-Oliveira y Rodil-Marzábal, 2019, quienes consideran que el tamaño y la antigüedad de la empresa se relacionan con las barreras de innovación. Sin embargo, Arza y López (2018) en un sentido parecido a este estudio presentan que el tamaño de la empresa no se relaciona con las barreras de la innovación en Argentina.

Por otra parte, en el sector manufacturero ecuatoriano, la percepción de obstáculos de la actividad empresarial no difiere entre si son empresas innovadoras o no, mientras que, en el caso argentino, existe diferencia en algunas barreras desde la percepción de los empresarios manufactureros innovadores y de los no innovadores.

Los resultados contribuyen a la literatura empírica sobre los obstáculos de la innovación de las empresas manufactureras en dos países latinoamericanos con diferentes perfiles de ciencia, tecnología e innovación y, sobre todo, en Ecuador, donde la evidencia empírica es escasa y con limitados estudios comparativos en materia de innovación.

La investigación pone de manifiesto la importancia del Estado y los hacedores de las políticas públicas en sus contextos específicos para que fomenten la cultura de la legalidad y la transparencia en los gobiernos de la región, con la finalidad de favorecer el desarrollo de los procesos de innovación y su desarrollo económico con entornos macroeconómicos estables y con mecanismos institucionales de apoyo flexibles para las empresas.

Algunos instrumentos de políticas dentro de los procesos de innovación orientados al sector de la manufactura pueden ser otorgar créditos fiscales para I+D, con el fin de mejorar la innovación tecnológica de productos y procesos, así como la cooperación regional entre países y empresas manufactureras para el desarrollo de la innovación a través de la transferencia de tecnología que esté más cerca del mercado. Todo esto dentro de instancias de diálogos transparentes entre el sector público y privado que favorezcan la innovación.

Respecto a las limitaciones del estudio, es importante aclarar que el tamaño de la muestra de las empresas del sector manufacturero de Ecuador es menor con respecto a la de Argentina; sin embargo, la información del Banco Mundial permitió comparar las limitaciones de la innovación de las empresas manufactureras, objeto del estudio, en dos países con diferente perfil de CTI. 
Una línea de investigación futura es analizar los sistemas nacionales de innovación de ambos países para identificar los elementos claves del ecosistema innovador y las funciones que cumplen las instituciones públicas que permitan su comparabilidad, además de un análisis exhaustivo de las herramientas de soporte a la innovación dentro de la actuación pública de sus gobiernos.

\section{Conflicto de intereses}

Las autoras declaran no tener ningún conflicto de intereses.

\section{Bibliografía}

Allard, G., Martinez, C. A. y Williams, C. (2012). Political instability, probusiness market reforms and their impacts on national systems of innovation. Research Policy, 41(3), 638-651. https://doi.org/10.1016/j.respol.2011.12.005

Álvarez, R. y Crespi, G. (2015). Heterogeneous effects of financial constraints on innovation: Evidence from Chile. Science and Public Policy, 42(5), 711-724. https://doi.org/10.1093/scipol/scu091

Arza, V. y López, E. (2018). Obstacles to innovation and firm size: A quantitative study for Argentina. Nueva York: Inter-American Development Bank. https://doi.org/10.18235/0001177

Banco Mundial (2014). Implementing enterprise surveys in Latin America and the Caribbean. Latin American and the Caribbean Series. Note N.o 1. Recuperado el día 10 de noviembre de 2020, de: https://n9.cl/4bner

Banco Mundial (2017). Enterprise Surveys. Recuperado el 3 de noviembre de 2017, de: https://n9.cl/1omyw

Barasa, L. (2018). Corruption, transaction costs, and innovation in Africa. African Journal of Science, Technology, Innovation and Development, 10(7), 811-821. https://doi.org/10.1080/20421338.2018.1519061

Bartels, F. L., Koria, R. y Andriano, L. (2016). Effectiveness and efficiency of national systems of innovation: A comparative analysis of Ghana and Kenya. African Journal of Science, Technology, Innovation and Development, 8(4), 343-356.

https://doi.org/10.1080/20421338.2015.1128037

Botric, V. y Božić, L. (2018). Human capital as barrier to innovation: Post-transition experience. International Journal of Innovation and Technology Management, 15(04), 1-17. https://doi.org/10.1142/s0219877018500335

Cecere, G., Corrocher, N. y Mancusi, M. L. (2018). Financial constraints and public funding of eco-innovation: Empirical evidence from European SMEs. Small Business Economics, 54, 285-302. https://doi.org/10.1007/s11187-018-0090-9

Coad, A., Pellegrino, G. y Savona, M. (2016). Barriers to innovation and firm productivity. Economics of Innovation and New Technology, 25(3), 321-334. https://doi.org/10.2139/ssrn.2742111

Crespi, G., Olivari, J. y Vargas, F. (2016). Productividad e innovación y la nueva economía de servicios en América Latina y el Caribe: retos e implicaciones de política. En J. Navarro y J. Olivari (Eds.), La política de innovación en América Latina y el Caribe: nuevos caminos (pp. 57-99). Washington: Banco Interamericano de Desarrollo. https://doi.org/10.18235/0000338

Cumming, D., Rui, O. y Wu, Y. (2016). Political instability, access to private debt, and innovation investment in China. Emerging Markets Review, 29, 68-81. https://doi.org/10.2139/ssrn.2632450

De León, I. y Fernández, J. (2016). El uso de la propiedad intelectual en el desarrollo de los mercados de innovación. En J. Navarro y J. Olivari (Eds.), La política de innovación en América Latina y el Caribe: nuevos caminos (pp. 199-229). Washington: Banco Interamericano de Desarrollo. https://doi.org/10.18235/0000338
de-Oliveira, F. y Rodil-Marzábal, Ó. (2019). Structural characteristics and organizational determinants as obstacles to innovation in small developing countries. Technological Forecasting and Social Change, 140, 306-314. https://doi.org/10.1016/j.techfore.2018.12.021

Dutrénit, G. (2012). Innovación para el desarrollo en América Latina: dónde estamos respecto a las masas críticas de capacidades. En I. Álvarez y C. Botella (Eds.), Innovación y Desarrollo: Retos para una Sociedad Global (pp.173-202). Madrid: Fundación Carolina/Siglo $\mathrm{XXI}$.

Edwards-Schachter, M., Castro-Martínez, E. y Fernández-de-Lucio, I. (2011). International co-operation between firms on innovation and R\&D: Empirical evidence from Argentina and Spain. Journal of Technology Management \& Innovation, 6(3), 126-147. https://doi.org/10.1787/118810438408

Foro Económico Mundial (2016). The Global Competitiveness Report 2016-2017. Geneva: WEF. Recuperado el 5 de noviembre de 2017, de: https://n9.cl/b5ypa

Goedhuys, M., Mohnen, P. y Taha, T. (2016). Corruption, innovation and firm growth: Firm-level evidence from Egypt and Tunisia. Eurasian Business Review, 6(3), 299-322. https://doi.org/10.1007/s40821-016-0062-4

Guaipatin, C. y Schwartz, L. (2014). Análisis del Sistema Nacional de innovación. Hacia la consolidación de una cultura innovadora. Washington: Banco Interamericano de Desarrollo.

Guercio, M., Vigier, H. y Briozzo, A. (2014). La forma legal y el acceso al financiamiento de las pymes industriales. DYNA Management, 2(1), 1-8. https://doi.org/10.6036/mn6946

Hoang-Nam, V. y Bao-Tram, H. (2019). Business environment and innovation persistence: The case of small-and mediumsized enterprises in Vietnam. Economics of Innovation and New Technology, 30(3), 239-261. https://doi.org/10.1080/10438599.2019.1689597

Kantis, H., Federico, J., Angelelli, P. e Ibarra-García, S. (2016). Business performance in young Latin American firms. En M. Grazzi y C. Pietrobelli (Eds.), Firm innovation and productivity in Latin America and the Caribbean (pp. 167-206). Washington: Banco Interamericano de Desarrollo.

Katz, R. y Galperín, H. (2013). La brecha de demanda: determinantes y políticas públicas. En R. Barrantes, V. Jordán y F. Rojas (Eds.), La evolución del paradigma digital en América Latina (pp. 33-68). Santiago de Chile: CEPAL, Naciones Unidas.

lammarino, S., Sanna-Randaccio, F. y Savona, M. (2009). The perception of obstacles to innovation. Foreign multinationals and domestic firms in Italy. Revue D'économie Industrielle, (125), 75-104. https://doi.org/10.4000/rei.3953

Iglesias-Sánchez, P., Jambrino-Maldonado, C. y De las Heras-Pedrosa, C. (2017). La innovación en la Pyme: Barreras y facilitadores. Revista de Estudios Regionales, (110), 99-131.

Lasio, V., Amaya, A., Zambrano, J. y Ordeñana, X. (2019). Global Entrepreneurship Monitor Ecuador 2019/2020. Guayaquil: ESPAE, Escuela de Negocios de la ESPOL.

Lachman, J. y López, A. (2019). Innovation obstacles in an emerging high tech sector. Management Research. Journal of the Iberoamerican Academy of Management, 4(14), 474-493. https://doi.org/10.1108/mrjiam-11-2018-0883

López, A., Niembro, A. y Ramos, D. (2017). Las empresas de servicios en América Latina: un estudio exploratorio sobre factores de competitividad internacional, obstáculos y políticas públicas. Tec Empresarial, 11(1), 7-22. https://doi.org/10.18845/te.v11i1.3099

Lundvall, B. A. (2016). The learning economy and the economics of hope. New York: Anthem Press.

Masino, S. (2015). Macroeconomic volatility, institutional instability and the incentive to innovate. Review of Development Economics, 19(1), 116-131. https://doi.org/10.1111/rode.12127

Mohan, P., Watson, P. y Strobl, E. (2016). Innovative activity in the Caribbean: Drivers, benefits, and obstacles. En Inter-American Development Bank, M. Grazzi y C. Pietrobelli (Eds.), Firm innovation 
and productivity in Latin America and the Caribbean (pp. 73-101). New York: Palgrave Macmillan.

https://doi.org/10.1057/978-1-349-58151-1_3

Mohnen, P. y Röller, L. (2005). Complementarities in innovation policy. European Economic Review, 49(6), 1431-1450. https://doi.org/10.1016/j.euroecorev.2003.12.003

Organización para la Cooperación y Desarrollo Económicos y Eurostat (2005). Manual de Oslo. Guía para la recogida e interpretación de datos sobre innovación (3.a ed.) (traducción española.). Grupo Tragsa.

Organización para la Cooperación y Desarrollo Económicos y Eurostat (2018). Oslo Manual 2018: Guidelines for collecting, reporting and using data on innovation, 4th Edition, The measurement of scientific, technological and innovation activities. Paris/Eurostat, Luxembourg: OECD Publishing. https://doi.org/10.1787/9789264304604-en

Paunov, C. (2016). Corruption's asymmetric impacts on firm innovation. Journal of Development Economics, 118, 216-231. https://doi.org/10.1016/j.jdeveco.2015.07.006

Pellegrino, G. (2015). Barriers to innovation: Can firm age help lower them? Working Papers 2015/3. Barcelona: Institut d'Economia de Barcelona (IEB). https://doi.org/10.2139/ssrn.2579801

Pellegrino, G. (2018). Barriers to innovation in young and mature firms. Journal of Evolutionary Economics, 28(1), 181-206. https://doi.org/10.1007/s00191-017-0538-0

Petelski, N., Milesi, D. y Verre, V. (2019). Public support to innovation: Impact on technological efforts in Argentine manufacturing firms. Economics of Innovation and New Technology, 29(1), 66-88. https://doi.org/10.1080/10438599.2019.1585672

Perry, G., Maloney, W., Arias, O., Fajnzylber, P., Mason, A. y SaavedraChanduvi, J. (2007). Informalidad: escape y exclusión. Estudios del Banco Mundial sobre América Latina y el Caribe. Washington: Banco Mundial.
Pirtea, M. G., Sipos, G. L. e lonescu, A. (2019). Does corruption affects business innovation? Insights from emerging countries. Journal of Business Economics and Management, 20(4), 715-733. https://doi.org/10.3846/jbem.2019.10160

Red de Indicadores de Ciencia y Tecnología Iberoamericana e Interamericana (2018). Indicadores de Insumo. Recuperado el 24 de septiembre de 2019, de: https://n9.cl/52waj

Sabato, J. y Botana, N. (2011). La ciencia y la tecnología en el desarrollo futuro de América Latina. En J. Sabato (Ed.), El pensamiento latinoamericano en la problemática ciencia-tecnología -desarrollodependencia (pp. 215-234). Buenos Aires: Ediciones Biblioteca Nacional.

Sanabria, S. (2008). El papel del transporte en el crecimiento económico colombiano en la segunda mitad del siglo XX. Apuntes del CENES, 27(46), 141-182.

Santiago, F., De Fuentes, C., Dutrénit, G. y Gras, N. (2016). What hinders innovation performance of services and manufacturing firms in Mexico? Economics of Innovation and New Technology, 26(3), 247-268. https://doi.org/10.1080/10438599.2016.1181297

Stendahl, M. y Roos, A. (2008). Antecedents and barriers to product innovation - a comparison between innovating and non-innovating strategic business units in the wood industry. Silva Fennica, 42(4), 659-681. https://doi.org/10.14214/sf.239

Xu, G. y Yano, G. (2016). How does anti-corruption affect corporate innovation? Evidence from recent anti-corruption efforts in China. Journal of Comparative Economics, 45(3), 1-23. https://doi.org/10.1016/j.jce.2016.10.001

Zamora, G. y Villamar, X. (2011). Caracterización de la pyme en la industria manufacturera del distrito metropolitano de quito. Quito: Publicaciones Pontificia Universidad Católica del Ecuador (PUCE). 